\title{
Exertional dyspnoea in pulmonary arterial hypertension
}

\author{
Daniel Dumitrescu ${ }^{1}$, Olivier Sitbon ${ }^{2,3,4}$, Jason Weatherald ${ }^{2,3,4,5}$ and \\ Luke S. Howard ${ }^{6,7}$ \\ Number 6 in the Series "Exertional dyspnoea" \\ Edited by Pierantonio Laveneziana and Piergiuseppe Agostoni
}

Affiliations: ${ }^{1}$ Klinik III für Innere Medizin, Herzzentrum der Universität zu Köln, Cologne, Germany. ${ }^{2}$ Univ. Paris-Sud, Faculté de Médecine, Université Paris-Saclay, Le Kremlin-Bicêtre, France. ${ }^{3}$ AP-HP, Hôpital Bicêtre, Service de Pneumologie, Le Kremlin-Bicêtre, France. ${ }^{4}$ INSERM UMR S 999, Hôpital Marie Lannelongue, Le Plessis Robinson, France. ${ }^{5}$ University of Calgary, Dept of Medicine, Division of Respirology, Calgary, AB, Canada. ${ }^{6}$ National Pulmonary Hypertension Service, Hammersmith Hospital, Imperial College Healthcare NHS Trust, London, UK. ${ }^{7}$ National Heart and Lung Institue, Imperial College London, London, UK.

Correspondence: Luke S. Howard, National Pulmonary Hypertension Service, Hammersmith Hospital, Imperial College Healthcare NHS Trust, Du Cane Road, London, W12 OHS, UK.

E-mail: l.howarddimperial.ac.uk

@ERSpublications

Dyspnoea is a complex integration of all the cardiopulmonary and systemic abnormalities in PAH http://ow.ly/D13W30dMDwJ

Cite this article as: Dumitrescu D, Sitbon O, Weatherald J, et al. Exertional dyspnoea in pulmonary arterial hypertension. Eur Respir Rev 2017; 26: 170039 [https://doi.org/10.1183/16000617.0039-2017].

ABSTRACT Dyspnoea is a principal presenting symptom in pulmonary arterial hypertension (PAH), and often the most distressing. The pathophysiology of PAH is relatively well understood, with the primary abnormality of pulmonary vascular disease resulting in a combination of impaired cardiac output on exercise and abnormal gas exchange, both contributing to increased ventilatory drive. However, increased ventilatory drive is not the sole explanation for the complex neurophysiological and neuropsychological symptom of dyspnoea, with other significant contributions from skeletal muscle reflexes, respiratory muscle function, and psychological and emotional status. In this review, we explore the physiological aspects of dyspnoea in $\mathrm{PAH}$, both in terms of the central cardiopulmonary abnormalities of $\mathrm{PAH}$ and the wider, systemic impact of PAH, and how these interact with common comorbidities. Finally, we discuss its relationship with disease severity.

Previous articles in this series: No. 1: Dubé B-P, Agostoni P, Laveneziana P. Exertional dyspnoea in chronic heart
failure: the role of the lung and respiratory mechanical factors. Eur Respir Rev 2016; 25: 317-332. No. 2: O’Donnell DE,
Elbehairy AF, Faisal A, et al. Exertional dyspnoea in COPD: the clinical utility of cardiopulmonary exercise testing. Eur
Respir Rev 2016; 25: 333-347. No. 3: Bernhardt V, Babb TG. Exertional dyspnoea in obesity. Eur Respir Rev 2016; 25:
487-495. No. 4: Bonini M, Fiorenzano G. Exertional dyspnoea in interstitial lung diseases: the clinical utility of
cardiopulmonary exercise testing. Eur Respir Rev 2017; 26: 160099. No. 5: Weatherald J, Lougheed MD, Taillé C, et al.
Mechanisms, measurement and management of exertional dyspnoea in asthma. Eur Respir Rev 2017; 26: 170015.

Received: April 012017 | Accepted after revision: June 032017

Support statement: Jason Weatherald is the recipient of a joint European Respiratory Society/Canadian Thoracic Society Long-Term Research Fellowship (LTRF 2015-4780).

Conflict of interest: Disclosures can be found alongside this article at err.ersjournals.com

Provenance: Commissioned article, peer reviewed.

Copyright OERS 2017. ERR articles are open access and distributed under the terms of the Creative Commons Attribution Non-Commercial Licence 4.0. 


\section{Introduction}

Pulmonary arterial hypertension $(\mathrm{PAH})$ is a primary disease of the pulmonary vasculature, which manifests physiologically principally through increased afterload on the right ventricle causing right ventricular failure, first through failure to augment cardiac output on exercise to match the exercise workload and subsequently, at rest, leading to progressive debilitating symptoms and death [1]. PAH is the quintessential cardiopulmonary disease, exerting its effects through both decreased cardiac output and impaired gas exchange, but also has far-reaching systemic effects on multiple organs through various mechanisms, including decreased tissue perfusion, inflammation and possible paraphenomena, such as autoimmunity [2].

On the face of it, dyspnoea may be considered to be a straightforward symptom, but this is by no means the case. The first descriptions of "cardiac dyspnoea" in the medical literature date back to 1895. LoomIs [3] proposed in a definition of this phenomenon that "the difficulty of breathing is due entirely to a cardiac condition" with "no organic changes within the lungs, bronchial tubes, or larynx to obstruct the entrance of air to the alveolar surfaces." Interestingly, he describes that autopsies of patients who fulfilled this definition and died showed that "usually the arrest is in the right heart, and as a result the blood is shut off from the pulmonary artery. The lungs under such circumstances will be not only free from congestion but more or less bloodless, while the other internal organs will be found intensely congested."

Most cases of PAH on an incremental exercise test are physiologically limited by cardiovascular function and not by lack of breathing reserve, yet the cardinal presenting feature of PAH is dyspnoea; once diagnosed and treated this is also perhaps the most dominant everyday symptom of PAH [4].

It is therefore important to consider that while patients may describe dyspnoea during everyday activities and often as the limiting factor in their activities, when undertaking an incremental test to maximum effort, other features come into play. Typical cardiovascular symptoms would include leg fatigue and presyncope or syncope. Although dyspnoea can be quantified by scores, such as the Dyspnoea-12 score [5], when the term "breathlessness" is used by PAH patients to describe their symptoms, it is a more complex summation of all the physiological disturbances in PAH. Dyspnoea may also be of prognostic relevance, with self-reported dyspnoea being identified as an independent predictor of the risk of death from cardiac causes in patients referred for cardiac stress testing [6].

There is a paucity of studies examining the specific mechanisms of dyspnoea in PAH. As a result, in this review, we have taken the approach to examine the individual components of exercise limitation in $\mathrm{PAH}$ and how they feed into dyspnoea, as well as describing how they contribute to the overall reduction in exercise capacity.

\section{Cardiovascular response to exercise in PAH}

The cardiovascular system responds to exercise in order to process the metabolic changes that occur with increased muscle work, including: 1) an increased, workload-dependent oxygen demand in the skeletal muscles, and 2) an increased generation of metabolic products affecting acid-base homeostasis, such as carbon dioxide.

According to the Fick principle, cardiac output can be calculated if oxygen uptake $\left(V^{\prime} \mathrm{O}_{2}\right)$ and the arterialmixed venous oxygen content difference $\left(\mathrm{Ca}-\mathrm{vO}_{2}\right)$ are known:

$$
\text { cardiac output }=V^{\prime} \mathrm{O}_{2} / \mathrm{Ca}-\mathrm{vO}_{2}
$$

In order to identify the cardiovascular components that determine an increase in $V^{\prime} \mathrm{O}_{2}$, the Fick equation may be rearranged and expanded:

$$
V^{\prime} \mathrm{O}_{2}=(\text { heart rate } \times \text { stroke volume }) \times \mathrm{Ca}_{\mathrm{a}-\mathrm{vO}_{2}}
$$

This equation shows that changes in $V^{\prime} \mathrm{O}_{2}$ are directly dependent on three parameters: stroke volume, heart rate and the $\mathrm{Ca}-\mathrm{vO}_{2}$. These parameters themselves reflect additional physiological adaptative mechanisms, with stroke volume depending on systolic and diastolic ventricular function and valvular competency, and heart rate depending on chronotropic competence and stimulation of adrenergic receptors. The $\mathrm{Ca}_{\mathrm{a}} \mathrm{vO}_{2}$ reflects oxygen transport capacity (haemoglobin) and peripheral oxygen extraction in the peripheral muscles. Therefore, $V^{\prime} \mathrm{O}_{2}$ is a comprehensive but nonspecific measure of cardiovascular and metabolic function and correlates with haemodynamic changes. However, this equation also demonstrates that any

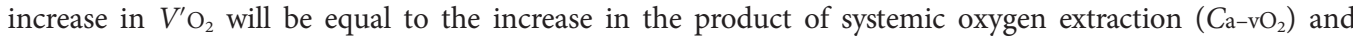
cardiac output (heart ratexstroke volume). Both mechanisms may be pathologically altered in PAH. 
The predominant cardiovascular abnormality of PAH on exercise is that of a failure to augment cardiac output on exercise due to increased right ventricular afterload, and this in turn is largely due to a failure to augment cardiac stroke volume [7]. Unlike in healthy subjects, where pulmonary vascular resistance falls on exercise due to pulmonary vascular recruitment and distensibility of the resistive vessels [8], the pulmonary vascular resistance does not fall in $\mathrm{PAH}$ to accommodate rises in cardiac output and pulmonary arterial pressure. Thus, increases in pulmonary arterial pressure on exercise reflect changes in cardiac output and stroke volume. Such a limitation in right ventricular contractile reserve during exercise may lead to impaired left ventricular filling during exercise and, as a consequence, exercise systemic blood pressure may not increase adequately, leading to presyncope and syncope in patients with advanced disease. In fact, several studies have been able to demonstrate that this aggregate of pathophysiological abnormalities contains relevant prognostic information. The absence of an adequate right ventricular contractile reserve [9] as well as the failure to increase systemic blood pressure during exercise [10] have been identified as predictors of poor prognosis, whereas greater increases in exercise cardiac output and systolic pulmonary arterial pressure identify better prognosis and response to therapy $[7,11]$.

Skeletal muscle dysfunction and severe, chronic deconditioning may be an underestimated problem in $\mathrm{PAH}$ patients [12]. With respect to the Fick equation, skeletal muscle function during exercise relates to $\mathrm{Ca}-\mathrm{vO}_{2}$, as remaining organ oxygen demand is assumed to be relatively constant during exercise, and an increase in oxygen extraction is mainly caused by increased skeletal muscle metabolism. However, oxygen extraction is difficult to measure in clinical practice, as it requires sampling of mixed venous blood. Therefore, a direct evaluation of skeletal muscle function in clinical practice, outside of studies, is difficult.

Only limited data are available regarding the $\mathrm{Ca}-\mathrm{vO}_{2}$ during exercise in pulmonary hypertension. In one study, maximum systemic oxygen extraction during exercise was impaired in $\mathrm{PAH}$, compared with pulmonary venous hypertension, suggesting that this may contribute to exercise intolerance [13]. However, this is in contrast to studies of heart failure (which would be expected to share many similarities with $\mathrm{PAH}$ ) that showed an increase in peak oxygen extraction similar to that found in highly trained athletes [14]. Further studies in heart failure have demonstrated that impaired diffusional transport of oxygen may be amenable to an exercise rehabilitation programme [15], but a recent detailed assessment of the exercise haemodynamic changes following a comprehensive exercise programme did not report on these aspects [16]. Impairment of muscle flow has not been evaluated in pulmonary hypertension, but may be an additional contributing factor, as previously demonstrated in heart failure patients $[15,17]$.

This combination of cardiac impairment and skeletal muscle dysfunction leads to impaired oxygen delivery and oxygen extraction, resulting in early anaerobiasis, and this in turn results in increased carbon dioxide production $\left(V^{\prime} \mathrm{CO}_{2}\right)$, necessitating an increase in alveolar ventilation. It is thus relatively straightforward to understand how these components of the "PAH syndrome" result in an increased ventilatory demand. However, the link to the sensation of dyspnoea is not so clear. While ventilation remains coupled to $V^{\prime} \mathrm{CO}_{2}$ in order to maintain $\mathrm{pH}$, as a result of early anaerobic metabolism, its relation to $V^{\prime} \mathrm{O}_{2}$ and work will become uncoupled. The sensation of ventilation being out of proportion to workload is potentially one of the mechanisms by which dyspnoea is perceived [18]. This may be mediated, at least in part, by afferent muscle reflexes themselves, the so-called "metaboreflex" and

FIGURE 1 Schematic representation of some of the inputs into the sensation of dyspnoea.

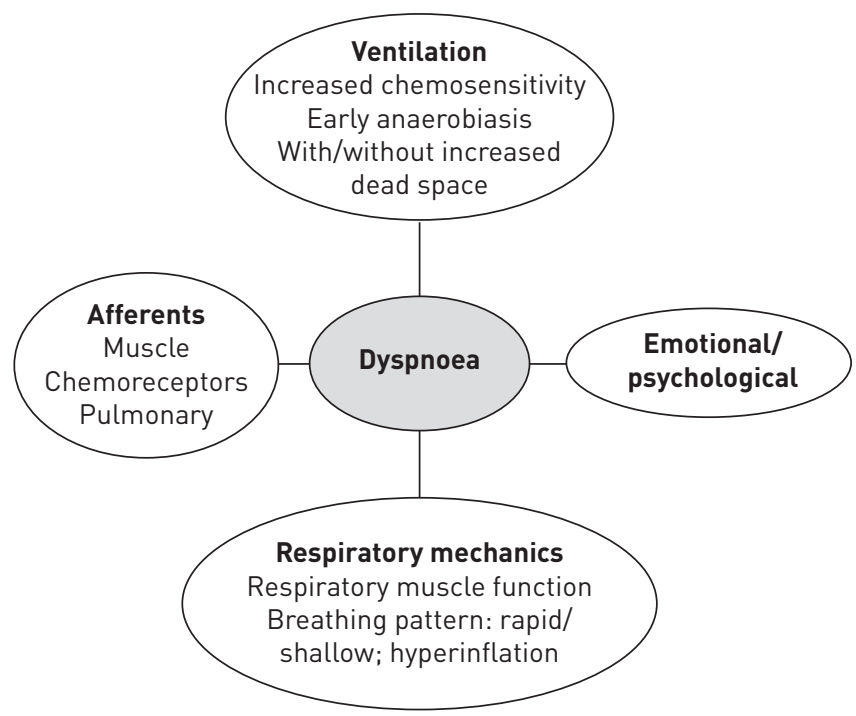


"mechanoreflex", which feed into central pathways regulating the cardiovascular and ventilatory responses to exercise as well as the sensation of effort [19]. These reflexes in turn integrate with other afferent inputs, e.g. chemoreceptors and respiratory afferents, to produce the sensation of dyspnoea (figure 1). This is beyond the scope of this review and has been reviewed in more detail elsewhere $[18,20]$.

Lastly, patients may experience myocardial ischaemia, further contributing to the symptoms of dyspnoea. Usually, this relates to an imbalance between oxygen supply and demand, but in rare circumstances pulmonary artery dilatation may cause a significant compression of the left coronary artery main stem (figure 2) and potentially lead to unexpected sudden death in precapillary pulmonary hypertension [21].

\section{Respiratory response to exercise in PAH}

The ventilatory response to exercise in PAH can be considered as an interaction between ventilatory demand, ventilatory pump function, breathing pattern and indirect inputs such as effort-output uncoupling and emotion. These are summarised in figure 1. In an incremental exercise test to maximal effort, the ventilatory demand, or more simply, ventilation, is considered in relation to $V^{\prime} \mathrm{CO}_{2}$ and is driven by a number of factors, all of which are altered in $\mathrm{PAH}$, resulting in ventilatory inefficiency. The relationship between minute ventilation $\left(V^{\prime} \mathrm{E}\right), V^{\prime} \mathrm{CO}_{2}$ and its other modifiying factors can be expressed as follows:

$$
V^{\prime} \mathrm{E}=V^{\prime} \mathrm{CO}_{2} \times 863 /\left(P_{\mathrm{aCO}} \times(1-V \mathrm{D} / V \mathrm{~T})\right)
$$

$\mathrm{PaCO}_{2}$ is the arterial partial pressure of carbon dioxide and $V \mathrm{D} / V \mathrm{~T}$ is the physiological dead space volume as a fraction of tidal volume. As a result of earlier anaerobiasis, $V^{\prime} \mathrm{CO}_{2}$ is increased earlier in exercise in $\mathrm{PAH}$ than in healthy individuals due to the buffering of lactic acid, leading to increased ventilatory demand at lower workloads:

$$
\text { Lactate }+\mathrm{H}^{+}+\mathrm{NaHCO}_{3} \leftrightarrow \mathrm{Na} \cdot \text { Lactate }+\mathrm{H}_{2} \mathrm{CO}_{3} \leftrightarrow \mathrm{Na} \cdot \text { Lactate }+\mathrm{H}_{2} \mathrm{O}+\mathrm{CO}_{2}
$$

In $\mathrm{PAH}, \mathrm{PaCO}_{2}$ is reduced due to chronic hyperventilation, which is thought to relate to heart failure and increased chemosensitivity [22-24]. This increases the ventilatory demand in relation to carbon dioxide output, already increased through early anaerobiasis. This is often referred to as ventilatory inefficiency and quantified as the ratio of $V^{\prime} \mathrm{E}$ to $V^{\prime} \mathrm{CO}_{2}$, expressed either as a slope relating the change of $V^{\prime} \mathrm{E}$ per unit change in $V^{\prime} \mathrm{CO}_{2}\left(V^{\prime} \mathrm{E} / V^{\prime} \mathrm{CO}_{2}\right.$ slope) or as a ratio at a fixed point in time (the ventilatory equivalent for $\mathrm{CO}_{2}$ $\left.\left(\mathrm{EqCO}_{2}\right)\right)$. While increased physiological dead space may be more relevant to the worse ventilatory inefficiency seen in chronic thromboembolic pulmonary hypertension (CTEPH) [25], it is likely that chronic hyperventilation, low $\mathrm{PaCO}_{2}$, is the main driver of ventilatory inefficiency in $\mathrm{PAH}$ [25], and indeed early computer modelling has suggested that hyperventilation may contribute to increased physiological dead space fraction [26]. Ventilatory inefficiency has also been shown to be worse in pulmonary veno-occlusive disease compared with $\mathrm{PAH}$, with commensurate worsening of dyspnoea. It is not clear whether this simply relates to disease severity or a distinct difference in gas exchange phenotype, although it is plausible that there is altered ventilation-perfusion mismatch and physiological dead space [27]. Further ventilatory inefficiency may occur in the setting of a right-to-left shunt through a patent foramen

FIGURE 2 Computed tomography coronary angiogram from a 43-year-old female patient with pulmonary arterial hypertension, showing compression of the left coronary artery main stem by a large pulmonary artery (PA) aneurysm. LCA: left coronary artery; AOV: aortic valve.

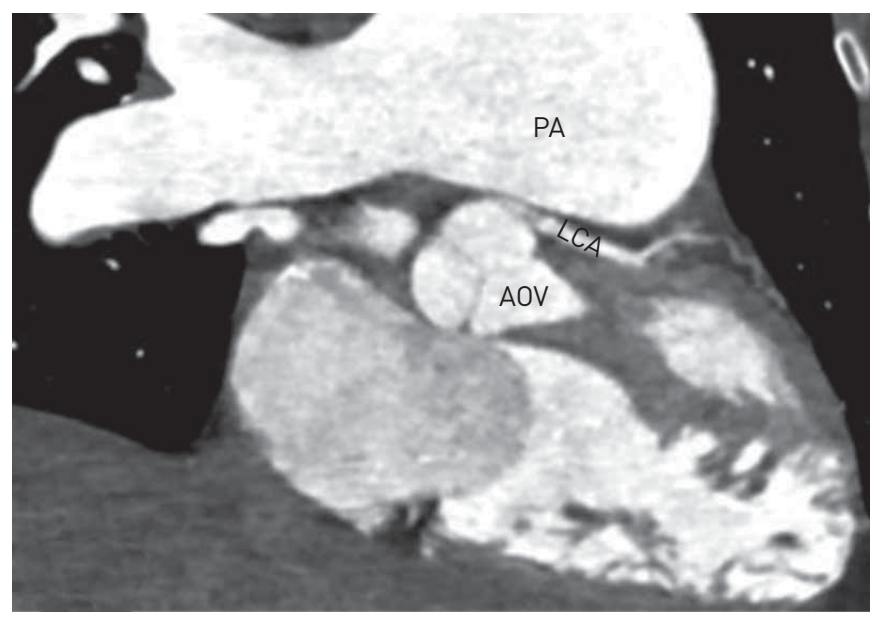


ovale [28] to maintain the carbon dioxide tension in admixed pulmonary venous and shunted blood, but in this scenario there will also be significant hypoxaemia, which will further stimulate ventilation through stimulation of the peripheral chemoreceptor reflex.

Beyond the respiratory compensation point in the incremental exercise test (i.e. the point at which the rate of production of lactic acid cannot be buffered by bicarbonate), $\mathrm{pH}$ falls, resulting in direct stimulation of ventilation and thus true hyperventilation and a further fall in $\mathrm{PaCO}_{2}$. Because the buffering capacity in $\mathrm{PAH}$ is reduced due to the compensatory metabolic acidosis related to chronic hyperventilation, this may result in an excessive increase in ventilation during intense exercise.

While most patients are limited by a lack of cardiovascular reserve at peak exercise in PAH, as evidenced by high peak respiratory exchange ratios and lactates at the end of exercise, rather than by a lack of breathing reserve, dyspnoea remains a cardinal symptom of pulmonary hypertension, even if not fundamentally the limiting factor on an incremental test. It is not entirely clear what drives dyspnoea, with one study showing that Borg dyspnoea ratings were higher at lower absolute levels of ventilation than in healthy subjects [29]. This same study demonstrated, however, that $60 \%$ of patients with PAH had reduced expiratory flows at low lung volumes and that dynamic hyperinflation accounted for $50-60 \%$ of the variance of Borg dyspnoea ratings during cycle ergometry [29].

More often than not, cycle ergometry is used to assess the cardiopulmonary response to incremental exercise, but there are important differences in gas exchange and dyspnoea between cycling and walking, with the latter most likely to reflect everyday symptoms. VALLI et al. [30] demonstrated that ventilatory inefficiency is considerably worse on walking than cycling, with higher dyspnoea at peak exercise, although absolute levels of exercise as assessed by oxygen consumption and $V^{\prime} \mathrm{CO}_{2}$ were lower. They suggested that this was related to worsened ventilation-perfusion matching, but also noted a more rapid and shallower breathing pattern during walking exercise. This highlights that while cycle ergometry may offer many advantages, such as standardisation of workload, it may not provide the best representation of patients' functional limitation.

Both increased ventilatory demand and dynamic hyperinflation place increased demand on the respiratory muscles. Unlike muscles, which may increase in strength/endurance with increased loading, there is mixed evidence of respiratory muscle weakness in pulmonary hypertension [12, 29, 31, 32]. Although this does not lead to exercise limitation, it is conceivable, although unproven, that this may contribute to the sensation of dyspnoea.

Finally, there is likely to be significant interplay between psychological status and dyspnoea. Although the breathing pattern in PAH has been shown to be rapid and shallow [29], frank breathing pattern disorders, such as hyperventilation, may coexist with $\mathrm{PAH}$, just as they may in other cardiorespiratory disorders. However, dyspnoea, as assessed by the Dyspnoea-12 questionnaire, shows stronger correlations with anxiety and depression scores than functional class or 6-min walking distance [5], highlighting the complex nature of dyspnoea and how it may be influenced by emotional and psychological health.

\section{Cardiopulmonary exercise physiology in relation to disease severity in PAH}

The severity of PAH can be categorised in terms of haemodynamic variables, functional capacity by New York Heart Association (NYHA) classification, or by the degree of impairment in peak $V^{\prime} \mathrm{O}_{2}$ during incremental cardiopulmonary exercise testing. With progressive severity of $\mathrm{PAH}$, interactions between the heart, lungs, peripheral muscle and autonomic nervous system become increasingly complex. Interestingly, no studies have specifically investigated the relationship between the haemodynamic severity of PAH and dyspnoea intensity during exercise testing. As expected, patients with worse NYHA functional class also have lower peak $V^{\prime} \mathrm{O}_{2}$, higher $V^{\prime} \mathrm{E} / V^{\prime} \mathrm{CO}_{2}$ slope and higher $\mathrm{EqCO}_{2}$, but the correlations between these variables and NYHA are modest, reinforcing the subjective and multifactorial nature of the NYHA classification [33]. Resting haemodynamic variables such as mean pulmonary arterial pressure, cardiac output and pulmonary vascular resistance also correlate with exercise capacity in terms of peak $V^{\prime} \mathrm{O}_{2}$ or NYHA functional class (figure 3), but a substantial portion of inter-individual variability in exercise capacity cannot be explained by the haemodynamic severity $[33,34]$. As such, patients with higher resting mean pulmonary arterial pressure tend to have lower peak $V^{\prime} \mathrm{O}_{2}$ and higher $V^{\prime} \mathrm{E} / V^{\prime} \mathrm{CO}_{2}$, but with considerable variation in ventilatory inefficiency between patients of comparable haemodynamic severity [34, 35].

As already discussed, among the major determinants of exercise capacity in PAH are 1) the ability to increase cardiac output during exercise to meet metabolic demand, 2) the ability of the pulmonary circulation to accommodate increased pulmonary blood flow, and 3) impaired skeletal muscle function. Recently, Hasler et al. [36] studied PAH patients undergoing supine exercise by cycle ergometry during right heart catheterisation, and found that the increase in cardiac index during exercise negatively correlated with disease severity and was a strong independent predictor of survival (hazard ratio 0.25 , 
FIGURE 3 Progressive physiological changes in pulmonary arterial hypertension. $\quad V^{\prime} E$ : minute ventilation; $V^{\prime} \mathrm{CO}_{2}$ : carbon dioxide production; PVR: pulmonary vascular resistance; $V^{\prime} \mathrm{O}_{2}$ : oxygen uptake; NYHA: New York Heart Association.

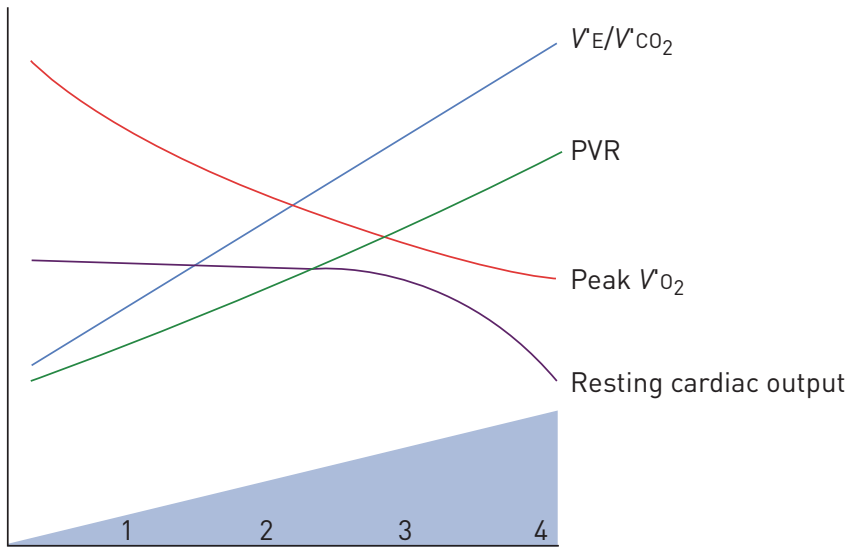

NYHA functional classification

$\mathrm{p}=0.04$ ). Patients with mild PAH (NYHA class I and II) were able increase cardiac output significantly during exercise [36], but even mildly symptomatic patients still have reduced exercise capacity compared to healthy individuals [33]. Because pulmonary vascular resistance is still elevated in mild PAH patients, there is a disproportionate increase in mean pulmonary arterial pressure as cardiac output rises.

One mechanism by which this may limit exercise capacity in mild PAH is through ventricular interaction. Pulmonary arterial and right ventricular pressures increase substantially during exercise, which can shift the interventricular septum in diastole, impairing left ventricular diastolic filling, stroke volume and cardiac output [37]. In contrast, patients with NYHA class III or IV symptoms have lower cardiac output and lower mixed venous oxygen content at rest, and demonstrate a diminished ability to increase cardiac output during exercise [36]. Several studies of PAH patients have shown a reduced ability to augment stroke volume during exercise, which necessitates an excessive increase in heart rate to increase cardiac output [38-40]. Consequently, the oxygen pulse $\left(V^{\prime} \mathrm{O}_{2} /\right.$ heart rate), which is an indirect estimate of stroke volume during cardiopulmonary exercise testing, is reduced in mild PAH patients in comparison to healthy individuals and is even further reduced in severe PAH patients [33].

Functional capacity and $V^{\prime} \mathrm{E} / V^{\prime} \mathrm{CO}_{2}$ in $\mathrm{PAH}$ are influenced by autonomic nervous system hyperactivity in addition to cardiac and gas exchange impairment $[41,42]$. For example, although $V \mathrm{D} / V \mathrm{~T}$ is elevated in patients with $\mathrm{PAH}$ and is associated with severity [25], high $V \mathrm{D} / V_{\mathrm{T}}$ explains only part of the increase in $V^{\prime} \mathrm{E} / V^{\prime} \mathrm{CO}_{2}$ and $\mathrm{EqCO}_{2}[25,35]$. Therefore, mechanisms other than increased dead space, such as enhanced chemosensitivity or hyperventilation due to a reduced $\mathrm{PaCO}_{2}$ set-point, contribute to the excessive ventilatory response and high $V^{\prime} \mathrm{E} / V^{\prime} \mathrm{CO}_{2}$ in severe $\mathrm{PAH}$. When right ventricular function and cardiac output are impaired, low mixed venous oxygen content and hypoxaemia are exaggerated during exercise. Thus, more severe impairment in the cardiovascular response to exercise leads to increased sympathetic drive and higher ventilatory response for a given metabolic demand (i.e. higher $V^{\prime} \mathrm{E} / V^{\prime} \mathrm{CO}_{2}$ ). This may explain why $V^{\prime} \mathrm{E} / V^{\prime} \mathrm{CO}_{2}$ not only reflects the severity of $\mathrm{PAH}$, but is also a powerful predictor of prognosis, as it integrates the function of the cardiac, respiratory and nervous systems [10]. While the $V^{\prime} \mathrm{E} / V^{\prime} \mathrm{CO}_{2}$ slope is usually elevated in the range of 35-45 in mild $\mathrm{PAH}$ patients [33], a $V^{\prime} \mathrm{E} / V^{\prime} \mathrm{CO}_{2}$ slope $>60$ (or EqCO $>54)$ is typically observed in severe patients, which portends a significantly increased risk of death [43, 44]. A low end-tidal carbon dioxide tension $\left(P \mathrm{ETCO}_{2}\right)$ and $\mathrm{PaCO}_{2}$ are also useful markers of PAH severity during exercise testing, as they reflect inefficient ventilation due to both high dead space and altered chemoreflex sensitivity with a lower $\mathrm{PaCO}_{2}$ set-point $[22,25,34]$. Idiopathic pulmonary arterial hypertension (IPAH) patients with low resting $\mathrm{PaCO}_{2}$ (those who hyperventilate at rest) have a lower cardiac index, lower mixed venous oxygen saturation and worse survival [22]. During exercise, the excessive ventilatory response seen in $\mathrm{PAH}$ patients further dilutes $\mathrm{PETCO}_{2}$, causing a progressive decline in $\mathrm{PETCO}_{2}$ during exercise, which is also proportional to the severity of disease [34].

\section{Exercise limitation phenotypes in different forms of pulmonary hypertension}

Pulmonary hypertension consists of a group of complex and heterogeneous diseases and, as such, there is no homogeneous pattern of exercise limitation among different groups of the 2013 pulmonary hypertension classification from the 5th World Symposium on Pulmonary Hypertension in Nice, France [45]. Publications on characteristic mechanisms or patterns of exercise intolerance and dyspnoea for different pulmonary hypertension forms are limited; however, there is a growing number of studies 
dedicated to exercise limitation in pulmonary hypertension subgroups. Because this review focuses mainly on $\mathrm{PAH}$, we shall only briefly discuss other forms of pulmonary hypertension in order to contrast with $\mathrm{PAH}$.

Patients with combined lung disease and heart disease often have a particularly severe phenotype, characterised by severe dyspnoea and impaired gas exchange. For example, in coexistent chronic obstructive pulmonary disease (COPD) and heart failure, there is a worsening of dead space ventilation, as indicated by a raised $V^{\prime} \mathrm{E} / V^{\prime} \mathrm{CO}_{2}$ slope intercept [46]. In pulmonary hypertension, however, the impact of coexisting lung disease is perhaps best illustrated in a detailed physiological study using invasive exercise haemodynamics in patients with COPD and different levels of severity of pulmonary hypertension [47]. Patients with mild and moderate pulmonary hypertension (i.e. somewhat more in proportion to their COPD severity) displayed ventilatory limitation with relatively preserved $V^{\prime} \mathrm{E} / V^{\prime} \mathrm{CO}_{2}$ relationships and hypercapnia at peak exercise indicative of respiratory mechanical limitation; however, patients with severe pulmonary hypertension behaved more like those with $\mathrm{PAH}$, showing high $V^{\prime} \mathrm{E} / V^{\prime} \mathrm{CO}_{2}$ slopes, hypocapnia and an exhausted circulatory reserve. Thus, despite a "double hit" of lung disease and severe pulmonary hypertension, this group of severe patients may be those whose symptoms of dyspnoea benefit from PAH therapies, although this has yet to be proven in randomised controlled studies. This study also serves to demonstrate that when PAH and mild coincidental lung disease coexist, such as may occur in scleroderma, the circulatory changes of PAH tend to dominate symptoms and exercise limitation.

CTEPH shares many similarities with PAH, but there are reasons to believe that they may differ in terms of the mechanisms of dyspnoea, in particular illustrated by the commonly encountered situation of chronic thromboembolic disease in the absence of pulmonary hypertension, which often results in significant symptoms of dyspnoea. Two studies have compared exercise gas exchange patterns in CTEPH and $\mathrm{PAH}[25,48]$. Both studies conclude that significant differences in gas exchange patterns exist, most probably due to a higher physiological $V \mathrm{D} / V \mathrm{~T}$ in $\mathrm{CTEPH}$ compared to $\mathrm{PAH}$, due to vascular obstruction by organised thrombosis. This may lead to a dissociation between pulmonary hypertension severity (in terms of the impact on right ventricular function) and gas exchange in CTEPH, unlike PAH [49], such that dyspnoea may be less reflective of prognosis in CTEPH than PAH [25].

\section{Impact of comorbidities on exercise limitation/breathlessness in PAH}

$\mathrm{PAH}$ often presents as an isolated condition, particularly in "classic" IPAH in young individuals, but may coexist with other conditions. A fine line exists between IPAH with coexisting lung disease, such as mild incidental emphysema or asthma, where IPAH would have presented whether the incidental lung disease had been present or not, and group 3 pulmonary hypertension, where pulmonary hypertension is considered secondary to lung disease and would not have existed without it. In this review, we consider the former scenario, where it would be anticipated that the interaction of minor parenchymal lung disease and IPAH would lead to worsened ventilatory inefficiency and hypoxaemia on exercise, leading to worsened dyspnoea relative to cardiovascular symptoms when compared with isolated IPAH. Furthermore, this is exacerbated where this is coupled with reduced lung volumes. Few published data exist, but this is a cardiopulmonary pathophysiological phenotype that we recognise in our laboratories, and is similar to that seen in severe pulmonary hypertension "out of proportion" to COPD [47].

Obesity places an additional burden on cardiopulmonary function [50]. There is an oxygen cost of unloaded exercise, largely due to the increased/hidden work of moving increased limb weight against no resistance [51]. In addition, it has been noted that otherwise healthy patients with obesity have a higher oxygen cost of breathing, which may relate to the increase in the work of breathing through altered respiratory mechanics, such as increased intrathoracic pressure and changes in airway resistance due to reduced functional residual capacity [50]. This may incur a small, yet relevant, oxygen steal during exercise. Although the increased work of breathing may not be clinically relevant in healthy obese subjects in producing worse dyspnoea, there is increased ventilatory demand in $\mathrm{PAH}$, and we can thus speculate that this may become more important.

Iron deficiency has recently been recognised as an important comorbidity of IPAH and is associated with worse prognosis and exercise capacity. It has been suggested that iron deficiency is not just a marker of severity, but independently contributes to poor prognosis and exercise function [52]. No studies have assessed the impact of iron replacement on prognosis, but two open-label protocols have demonstrated improved aerobic capacity following intravenous iron, most probably due to improved skeletal muscle mitochondrial oxidative capacity [53, 54]. Data were not reported on dyspnoea specifically, but improvements were seen in both studies in quality of life as assessed by the SF-36 score (36-item Short-Form Health Survey).

It is debatable whether skeletal muscle, including both the peripheral locomotor groups and respiratory muscles, is considered a comorbidity of PAH or an integral part of the "PAH syndrome". In IPAH, as well 
as other forms of pulmonary hypertension isolated to the cardiopulmonary system, such as CTEPH, there are many studies documenting impaired skeletal muscle function. Clearly, in other forms of PAH that are part of a more formal systemic syndrome such as the connective tissue diseases, myopathy may be more severe, sometimes requiring specific therapy. In these cases, dissociating primary cardiopulmonary limitation from muscle disease is important, not only to make the appropriate diagnosis, but also to maximise the opportunity to treat muscle disease and to avoid overtreatment of pulmonary hypertension. Primary muscle disease may often present with muscle pain on exercise, but can also contribute to dyspnoea through early anaerobiasis, thus increasing ventilatory demand.

\section{Limitations}

Although exercise dyspnoea is a well-known and cardinal symptom of PAH, its complex multifactorial origin makes it difficult to understand and relate back to measurable pathophysiological mechanisms. The best measurement tools we have derive from exercise testing, but dyspnoea scores and questionnaires only provide us with crude mechanistic insight. Objective measurement of dyspnoea potentially requires more sophisticated instruments such as functional neurological imaging. Therapies targeting dyspnoea directly are frequently used at the end of life in palliative care settings, but none has been studied in less advanced disease, and while it may be feasible to do this, without better understanding the mechanisms and pathophysiological surrogates of dyspnoea, these therapeutic areas will be hard to explore.

\section{Conclusion}

Dyspnoea is the most common and often most debilitating symptom of $\mathrm{PAH}$, and while the physiology of $\mathrm{PAH}$ is well characterised, dyspnoea itself remains elusively misunderstood. Treatments that target PAH directly lead to improvements in dyspnoea, but it remains possible that opportunities exist to target dyspnoea directly without having an impact on the underlying disease process. Unless dyspnoea is better understood, these opportunities are likely to remain underexplored.

\section{References}

1 Vonk-Noordegraaf A, Haddad F, Chin KM, et al. Right heart adaptation to pulmonary arterial hypertension: physiology and pathobiology. J Am Coll Cardiol 2013; 62: Suppl. 25, D22-D33.

2 Kherbeck N, Tamby MC, Bussone G, et al. The role of inflammation and autoimmunity in the pathophysiology of pulmonary arterial hypertension. Clin Rev Allergy Immunol 2013; 44: 31-38.

3 Loomis AL. Cardiac dyspnoea. Trans Am Climatol Assoc 1895; 10: 114-120.

4 Guillevin L, Armstrong I, Aldrighetti R, et al. Understanding the impact of pulmonary arterial hypertension on patients' and carers' lives. Eur Respir Rev 2013; 22: 535-542.

5 Yorke J, Armstrong I. The assessment of breathlessness in pulmonary arterial hypertension: reliability and validity of the Dyspnoea-12. Eur J Cardiovasc Nurs 2014; 13: 506-514.

6 Abidov A, Rozanski A, Hachamovitch R, et al. Prognostic significance of dyspnea in patients referred for cardiac stress testing. N Engl J Med 2005; 353: 1889-1898.

7 Chaouat A, Sitbon O, Mercy M, et al. Prognostic value of exercise pulmonary haemodynamics in pulmonary arterial hypertension. Eur Respir J 2014; 44: 704-713.

8 Naeije R, Vanderpool R, Dhakal BP, et al. Exercise-induced pulmonary hypertension: physiological basis and methodological concerns. Am J Respir Crit Care Med 2013; 187: 576-583.

9 Grunig E, Tiede H, Enyimayew EO, et al. Assessment and prognostic relevance of right ventricular contractile reserve in patients with severe pulmonary hypertension. Circulation 2013; 128: 2005-2015.

10 Wensel R, Opitz CF, Anker SD, et al. Assessment of survival in patients with primary pulmonary hypertension: importance of cardiopulmonary exercise testing. Circulation 2002; 106: 319-324.

11 Blumberg FC, Arzt M, Lange T, et al. Impact of right ventricular reserve on exercise capacity and survival in patients with pulmonary hypertension. Eur J Heart Fail 2013; 15: 771-775.

12 Manders E, Rain S, Bogaard HJ, et al. The striated muscles in pulmonary arterial hypertension: adaptations beyond the right ventricle. Eur Respir J 2015; 46: 832-842.

13 Tolle J, Waxman A, Systrom D. Impaired systemic oxygen extraction at maximum exercise in pulmonary hypertension. Med Sci Sports Exerc 2008; 40: 3-8.

14 Katz SD, Maskin C, Jondeau G, et al. Near-maximal fractional oxygen extraction by active skeletal muscle in patients with chronic heart failure. J Appl Physiol 2000; 88: 2138-2142.

15 Esposito F, Reese V, Shabetai R, et al. Isolated quadriceps training increases maximal exercise capacity in chronic heart failure: the role of skeletal muscle convective and diffusive oxygen transport. J Am Coll Cardiol 2011; 58: 1353-1362.

16 Ehlken N, Lichtblau M, Klose H, et al. Exercise training improves peak oxygen consumption and haemodynamics in patients with severe pulmonary arterial hypertension and inoperable chronic thrombo-embolic pulmonary hypertension: a prospective, randomized, controlled trial. Eur Heart J 2016; 37: 35-44.

17 Sullivan MJ, Knight JD, Higginbotham MB, et al. Relation between central and peripheral hemodynamics during exercise in patients with chronic heart failure. Muscle blood flow is reduced with maintenance of arterial perfusion pressure. Circulation 1989; 80: 769-781.

18 Laviolette L, Laveneziana P. Dyspnoea: a multidimensional and multidisciplinary approach. Eur Respir J 2014; 43: 1750-1762.

19 Piepoli MF, Dimopoulos K, Concu A, et al. Cardiovascular and ventilatory control during exercise in chronic heart failure: role of muscle reflexes. Int J Cardiol 2008; 130: 3-10. 

mechanisms, assessment, and management of dyspnea. Am J Respir Crit Care Med 2012; 185: 435-452.

21 Zylkowska J, Kurzyna M, Florczyk M, et al. Pulmonary artery dilatation correlates with the risk of unexpected death in chronic arterial or thromboembolic pulmonary hypertension. Chest 2012; 142: 1406-1416.

22 Hoeper MM, Pletz MW, Golpon H, et al. Prognostic value of blood gas analyses in patients with idiopathic pulmonary arterial hypertension. Eur Respir J 2007; 29: 944-950.

23 Tumminello G, Guazzi M, Lancellotti P, et al. Exercise ventilation inefficiency in heart failure: pathophysiological and clinical significance. Eur Heart J 2007; 28: 673-678.

24 Raffestin B, Leroy M. Clinical relevance of autonomic nervous system disturbances in pulmonary arterial hypertension. Eur Respir J 2010; 35: 704-705.

25 Zhai Z, Murphy K, Tighe $\mathrm{H}$, et al. Differences in ventilatory inefficiency between pulmonary arterial hypertension and chronic thromboembolic pulmonary hypertension. Chest 2011; 140: 1284-1291.

26 West JB. Ventilation-perfusion inequality and overall gas exchange in computer models of the lung. Respir Physiol 1969; 7: 88-110.

27 Laveneziana P, Montani D, Dorfmüller P, et al. Mechanisms of exertional dyspnoea in pulmonary veno-occlusive disease with EIF2AK4 mutations. Eur Respir J 2014; 44: 1069-1072.

28 Sun XG, Hansen JE, Oudiz RJ, et al. Gas exchange detection of exercise-induced right-to-left shunt in patients with primary pulmonary hypertension. Circulation 2002; 105: 54-60.

29 Laveneziana P, Humbert M, Godinas L, et al. Inspiratory muscle function, dynamic hyperinflation and exertional dyspnoea in pulmonary arterial hypertension. Eur Respir J 2015; 45: 1495-1498.

30 Valli G, Vizza CD, Onorati P, et al. Pathophysiological adaptations to walking and cycling in primary pulmonary hypertension. Eur J Appl Physiol 2008; 102: 417-424.

31 de Man FS, van Hees HW, Handoko ML, et al. Diaphragm muscle fiber weakness in pulmonary hypertension. Am J Respir Crit Care Med 2011; 183: 1411-1418.

32 Manders E, Bonta PI, Kloek JJ, et al. Reduced force of diaphragm muscle fibers in patients with chronic thromboembolic pulmonary hypertension. Am J Physiol Lung Cell Mol Physiol 2016; 311: L20- L28.

33 Sun XG, Hansen JE, Oudiz RJ, et al. Exercise pathophysiology in patients with primary pulmonary hypertension. Circulation 2001; 104: 429-435.

34 Yasunobu Y, Oudiz RJ, Sun XG, et al. End-tidal $\mathrm{PCO}_{2}$ abnormality and exercise limitation in patients with primary pulmonary hypertension. Chest 2005; 127: 1637-1646.

35 Reybrouck T, Mertens L, Schulze-Neick I, et al. Ventilatory inefficiency for carbon dioxide during exercise in patients with pulmonary hypertension. Clin Physiol 1998; 18: 337-344.

36 Hasler ED, Müller-Mottet S, Furian M, et al. Pressure-flow during exercise catheterization predicts survival in pulmonary hypertension. Chest 2016; 150: 57-67.

37 Kasner M, Westermann D, Steendijk P, et al. Left ventricular dysfunction induced by nonsevere idiopathic pulmonary arterial hypertension: a pressure-volume relationship study. Am J Respir Crit Care Med 2012; 186: 181-189.

38 Chemla D, Castelain V, Hoette S, et al. Strong linear relationship between heart rate and mean pulmonary artery pressure in exercising patients with severe precapillary pulmonary hypertension. Am J Physiol Heart Circ Physiol 2013; 305: H769-H777.

39 Holverda S, Gan CT, Marcus JT, et al. Impaired stroke volume response to exercise in pulmonary arteria hypertension. J Am Coll Cardiol 2006; 47: 1732-1733.

40 Groepenhoff $\mathrm{H}$, Westerhof $\mathrm{N}$, Jacobs W, et al. Exercise stroke volume and heart rate response differ in right and left heart failure. Eur J Heart Fail 2010; 12: 716-720.

41 Ciarka A, Doan V, Velez-Roa S, et al. Prognostic significance of sympathetic nervous system activation in pulmonary arterial hypertension. Am J Respir Crit Care Med 2010; 181: 1269-1275.

42 Velez-Roa S, Ciarka A, Najem B, et al. Increased sympathetic nerve activity in pulmonary artery hypertension. Circulation 2004; 110: 1308-1312.

43 Schwaiblmair M, Faul C, von Scheidt W, et al. Ventilatory efficiency testing as prognostic value in patients with pulmonary hypertension. BMC Pulm Med 2012; 12: 23.

44 Deboeck G, Scoditti C, Huez S, et al. Exercise testing to predict outcome in idiopathic versus associated pulmonary arterial hypertension. Eur Respir J 2012; 40: 1410-1419.

45 Simonneau G, Gatzoulis MA, Adatia I, et al. Updated clinical classification of pulmonary hypertension. $J$ Am Coll Cardiol 2013; 62: Suppl. 25, D34-D41.

46 Apostolo A, Laveneziana $\mathrm{P}$, Palange $\mathrm{P}$, et al. Impact of chronic obstructive pulmonary disease on exercise ventilatory efficiency in heart failure. Int J Cardiol 2015; 189: 134-140.

47 Boerrigter BG, Bogaard HJ, Trip P, et al. Ventilatory and cardiocirculatory exercise profiles in COPD: the role of pulmonary hypertension. Chest 2012; 142: 1166-1174.

48 Scheidl SJ, Englisch C, Kovacs G, et al. Diagnosis of CTEPH versus IPAH using capillary to end-tidal carbon dioxide gradients. Eur Respir J 2012; 39: 119-124.

49 Rehman MB, Howard LS, Christiaens LP, et al. Resting right ventricular function is associated with exercise performance in PAH, but not in CTEPH. Eur Heart J Cardiovasc Imaging 2017; in press [https://doi.org/10.1093/ ehjci/jex002].

50 Parameswaran K, Todd DC, Soth M. Altered respiratory physiology in obesity. Can Respir I 2006; 13: $203-210$.

51 Salvadori A, Fanari P, Fontana M, et al. Oxygen uptake and cardiac performance in obese and normal subjects during exercise. Respiration 1999; 66: 25-33.

52 Rhodes CJ, Howard LS, Busbridge M, et al. Iron deficiency and raised hepcidin in idiopathic pulmonary arterial hypertension: clinical prevalence, outcomes, and mechanistic insights. J Am Coll Cardiol 2011; 58: 300-309.

53 Ruiter G, Manders E, Happe CM, et al. Intravenous iron therapy in patients with idiopathic pulmonary arterial hypertension and iron deficiency. Pulm Circ 2015; 5: 466-472.

54 Viethen T, Gerhardt F, Dumitrescu D, et al. Ferric carboxymaltose improves exercise capacity and quality of life in patients with pulmonary arterial hypertension and iron deficiency: a pilot study. Int J Cardiol 2014; 175: 233-239. 